\title{
Contemporary tectonic stress field in China*
}

\author{
Yongge Wan ${ }^{1,2, *}$ \\ ${ }^{1}$ Institute of Disaster-Prevention Science and Technology, Yanjiao, Sanhe 065201, China \\ ${ }^{2}$ Institute of Geophysics, China Earthquake Administration, Beijing 100081, China
}

\begin{abstract}
The contemporary tectonic stress field in China is obtained on the basis of Chinese stress field database and Harvard CMT catalogue. Result of the inverted tectonic stresses shows that the maximum principal stress axis strikes nearly north-south direction in the west part of Tibet plateau, ENE direction in North China. In Central China, its strikes show a radiated pattern, i.e., NNE in north part and NNW in south part. The detailed stress field parameters of nearly whole China are given and can be used in geodynamic stress field simulation and earthquake prediction.
\end{abstract}

Key words: tectonic stress field; focal mechanism; stress measurement

CLC number: P315.72 7 Document code: A

\section{Introduction}

Study of tectonic stress field, a major branch of Earth science, plays an important role in the studies of geodynamics. The World Stress Map Plan started in 1980s was led by M. L. Zoback. Lots of scientists participated in this plan. The plan collected global tectonic stress measurements and research results to establish global stress database. The world stress map was edited based on the global stress database. The world stress map reflects feature of global lithosphere stress field both in total and in subareas, and thus can explain the tectonic stress interaction in lithosphere (Zoback, 1992).

Tectonic stress field study has achieved significant development in China. In the early of $1970 \mathrm{~s}$, Li et al (1973) studied the stress field near a seismic station by synthetic first motion pattern of multi earthquakes (Aki, 1966). Xu et al (1983) extended this idea to stress determination by using multi- micro-earthquake and multi-station, further applied it to North China area. This method was also used to determine the tectonic stress field around Ordos block (Xue and Yan, 1984), East China (Wang and Xu, 1985) and Southwest China (Xu et al, 1987). Xu et al (1992) obtained the fundamental features of stress field in Chinese mainland by

\footnotetext{
* Received 9 April 2010; accepted in revised form 12 June 2010; published 10 August 2010.

* Corresponding author. e-mail: wanyg217217@vip.sina.com

(C) The Seismological Society of China and Springer-Verlag Berlin Heidelberg 2010
}

summarizing the stress field directions of previous studies, which reflects the close relationship between the motion of each block and that of the adjacent blocks. According to focal mechanism data and deep hole breakouts, Xu (2001) gave the present-day tectonic stress map for eastern Asia region. The maps of orientation of principal stress axes show that, apart from the strong influence of the collision between the Indian plate and the Eurasian plate, the present-day tectonic stress in eastern Asia is significantly affected by the back-arc extension of the subduction zones. The joint effect of the continental collision at Himalaya arc and back-arc extension in Myanmar arc region may be responsible for the remarkable rotation of principal stress orientations in southeastern part of Tibet plateau.

Another stress field determination method is using fault slip data and focal mechanism data (Angelier, 1979; Gephart and Forsyth, 1984; Michael, 1987). Based on stress determination method of Angelier (1979), Xu and Ge (1984) improved it and applied it to the 1931 M8.0 Fuyun, Xinjiang, earthquake. Using the similar method, the tectonic stress field in Southwest China (Xie et al, 1993; Cui and Xie, 1999) and in Guangdong and its adjacent areas (Kang et al, 2008) have been obtained. By using focal mechanism data in Chinese mainland, Du and Shao (1999) derived the tectonic stress field and principal stress ratio.

Nevertheless, neither $\mathrm{Xu}$ et al (1992) nor $\mathrm{Du}$ and Shao (1999) could cover their stress analysis on the whole China. For example, their stress analysis did not 
cover most of Tarim basin, Ningxia Hui autonomous region, most part of Inner Mongolia autonomous region, Hubei and Hunan provinces, etc. However, the tectonic stress field in whole China is needed in some geodynamic and earthquake prediction study. For example, the load/unload response ratio method used to earthquake prediction needs to know the tectonic stress field in advance (Peng et al, 2000; Wan, 2004). In recent years, accumulation of stress measurement, fault slip measurement and focal mechanism data, especially establishment of crustal stress database in China and its adjacent areas (Xie et al, 2003), lays a solid foundation for the study on determination of Chinese tectonic stress field. By using this database, Xie et al (2004) summarized the fundamental features of Chinese tectonic stress field and divided the Chinese mainland into different tectonic stress blocks. In this study, we will divide the whole China into $2^{\circ} \times 2^{\circ}$ subregions and determine the tectonic stress direction and stress ratio in each subregion by using this database and CMT catalogue from 1976 to 2005 .

\section{Tectonic stress inversion method}

Studying the state of stress in the Earth's crust and upper mantle is helpful in understanding plate motion and regional deformation (Hardebeck and Hauksson, 2001). Earthquake focal mechanisms are indicators of stress; thus, we will use earthquake focal mechanisms to detect stress state that cannot be directly measured. Several authors have proposed methods to determine orientations of stress axes of seismotectonic regime in spite of complicated tectonic settings (e.g., Gephart and Forsyth, 1984; Michael, 1984; Angelier, 1989; Horiuchi et al, 1995). For tectonic stress inversion is a nonlinear problem, Michael's method linearly determines the stress tensor by using least squares method and has a probability to trap in local minimum. So, in this paper, the focal mechanism stress inversion (short for FMSI) (Gephart and Forsyth, 1984) program by grid searching stress field parameters is used to determine the orientations of principal stress axes in China.

FMSI method has three basic assumptions (Gephart and Forsyth, 1984; Gephart, 1990): (1) slip on the fault plane occurs in the direction of resolved shear stress, (2) stress orientation is uniform in the calculated area, and (3) earthquakes are shear dislocations and can occur on preexisting faults. The FMSI method uses a grid search over stress field parameter space to find the best-fitting model that minimizes the average of the individual misfits between possible models and real data (Gephart and Forsyth, 1984; Gephart, 1990).

In FMSI, the individual misfit calculated for each earthquake is defined as the least rotation angle about any axis of general orientation which is needed to match the observed slip direction with one consistent with a given stress model (Gephart and Forsyth, 1984). We obtained the azimuths and plunges of three principal stresses axes $\sigma_{1}, \sigma_{2}$ and $\sigma_{3}\left(\sigma_{1} \geq \sigma_{2} \geq \sigma_{3}\right)$ and the ratio $R=$ $\left(\sigma_{2}-\sigma_{1}\right) /\left(\sigma_{3}-\sigma_{1}\right)(0 \leq R \leq 1)$ by the best-fitting model. This may help us to distinguish the stress filed type.

There are four stress parameters $\left(\sigma_{1}, \sigma_{2}, \sigma_{3}\right.$ and $\left.R\right)$ in the FMSI inversion algorithm, and the minimum number of events used to inversion is four. Moreover, diverse data set can give better constrains to find out the suitable stress tensor orientation. For above reasons, we used all the earthquake focal mechanisms within each data set to obtain an average local stress field without separating fault types in a region.

The procedure of FMSI to determine best-fitting stress model is as follows. We first perform a coarse initial grid search (with $10^{\circ}$ spacing in stress orientations) covering the whole range of possible models for each data set by the approximate FMSI method (short for FMSIA, Gephart, 1990). We then take the best resulting stress model as a starting model to perform a fine grid search (with $5^{\circ}$ spacing in stress orientations) by the exact FMSI method (short for FMSIE, Gephart, 1990).

In FMSI, the size of the average misfit corresponding to the best fitting stress model could be an indicator of the homogeneity degree of stress. According to a series of tests carried out by Wyss et al (1992) and Gillard et al (1996), for the real earthquake, focal mechanisms with errors of $15^{\circ}$ (average of the uncertainties in strike, dip and rake) cannot obtain the average misfit of the stress inversion larger than $6^{\circ}$, thus the average misfit smaller than $6^{\circ}$ may represent a homogeneous stress field. In contrast, the average misfit larger than $9^{\circ}$ could be attributed to heterogeneity of stress. In the case of average misfit in the range between $6^{\circ}$ and $9^{\circ}$, the stress solution is acceptable, but may reflect some heterogeneity (e.g., Wyss and Lu, 1995; Lu et al, 1997).

\section{Data}

The data used in Chinese tectonic field determination include: (1) 918 focal mechanism data from 1920 to 2003 determined by Chinese scholars, (2) 240 fault stria- 
tion data from Quaternary fault slip measurements, (3) 72 stress relief data with all three axis directions at measured depth range of 50-363 $\mathrm{m}$, and (4) 7 hydraulic fracturing stress data with all three axis directions at measured depth range from $400 \mathrm{~m}$ to $1620 \mathrm{~m}$. All the above data are from database of crustal stress in China and its adjacent areas (Xie et al, 2003). We also search the focal mechanism data from Harvard CMT solutions for the earthquakes in 1976-2005, which are not overlapped with the 918 focal mechanism data during 1920-2003 (Xie et al, 2003).

We divide the whole China into $2^{\circ} \times 2^{\circ}$ grids. In order to cover the whole study region and get the smoothing stress field, we select the data within the square areas of $5^{\circ} \times 5^{\circ}$ with the center of grid point. The stress field is smoothed by repeated selection of the focal mechanism data at different grid point, which looks more reasonable for stress field continuation. For the crust stress field has a probability different from the mantle stress field, we only select the data with depth less than $60 \mathrm{~km}$. We cannot invert stress field in the areas less than four focal mechanism data, and use the stress direction determined by composite focal mecha- nism solution of Wang and Xu (1985) instead (Figures 1 and 2 quivers without color filled).

\section{Results}

Basing on the data above mentioned, we get the stress field nearly covering the whole China (Figures 1 and 2) by the stress field determination method (Gephart and Forsyth, 1984; Gephart, 1990) and the results are listed in Table 1. From Figure 1, we can see that the bigger misfit angles are distributed in east part of Tibet plateau, Tianshan and its west area, Taiwan and south-east costal region of China, showing larger heterogeneity of stress field (e.g., Wyss and Lu, 1995; Lu and Wyss, 1996; Lu et al, 1997). But the continuity of stress field with other areas shows the overall pattern may be accepted. From Figure 2, we can see larger number of focal mechanisms used in the inversion in Taiwan region, south-east coastal region of China, west to Tianshan region and central region of the Tibet plateau, which shows more constraint to these stress field results.

Table 1 Results of stress field inverted in this study

\begin{tabular}{|c|c|c|c|c|c|c|c|c|c|c|}
\hline \multirow{2}{*}{$\begin{array}{l}\text { Lat. } \\
{ }^{\circ} \mathrm{N}\end{array}$} & \multirow{2}{*}{$\begin{array}{l}\text { Long. } \\
{ }^{\circ} \mathrm{E}\end{array}$} & \multicolumn{2}{|c|}{$\sigma_{1}$ axis } & \multicolumn{2}{|c|}{$\sigma_{2}$ axis } & \multicolumn{2}{|c|}{$\sigma_{3}$ axis } & \multirow{2}{*}{$R$} & \multirow{2}{*}{$\begin{array}{c}\text { Misfit angle } \\
/^{\circ}\end{array}$} & \multirow{2}{*}{$\begin{array}{l}\text { Number of } \\
\text { earthquakes }\end{array}$} \\
\hline & & $\mathrm{Az} /{ }^{\circ}$ & $\mathrm{Pl} /{ }^{\circ}$ & $\mathrm{Az} /^{\circ}$ & $\mathrm{P} 1{ }^{\circ}$ & $\mathrm{Az} /{ }^{\circ}$ & $\mathrm{Pl} /{ }^{\circ}$ & & & \\
\hline 22 & 99 & 212 & 7 & 97 & 73 & 304 & 15 & 0.45 & 6.926 & 78 \\
\hline 22 & 101 & 194 & 26 & 42 & 60 & 290 & 12 & 0.25 & 8.633 & 69 \\
\hline 22 & 103 & 354 & 26 & 145 & 60 & 258 & 12 & 0.25 & 5.129 & 48 \\
\hline 22 & 105 & 344 & 26 & 144 & 62 & 250 & 8 & 0.30 & 3.931 & 27 \\
\hline 22 & 107 & 337 & 25 & 138 & 63 & 243 & 7 & 0.40 & 1.550 & 10 \\
\hline 22 & 109 & 330 & 4 & 151 & 85 & 61 & 0 & & & 0 \\
\hline 22 & 111 & 330 & 4 & 151 & 85 & 61 & 0 & & & 0 \\
\hline 22 & 113 & 112 & 12 & 21 & 4 & 272 & 77 & 0.55 & 1.960 & 4 \\
\hline 22 & 115 & 84 & 17 & 298 & 69 & 177 & 11 & 0.20 & 4.012 & 11 \\
\hline 24 & 99 & 190 & 35 & 36 & 52 & 289 & 13 & 0.35 & 9.034 & 90 \\
\hline 24 & 101 & 18 & 9 & 257 & 72 & 110 & 15 & 0.65 & 10.973 & 81 \\
\hline 24 & 103 & 359 & 10 & 127 & 74 & 267 & 12 & 0.55 & 7.574 & 62 \\
\hline 24 & 105 & 339 & 30 & 141 & 58 & 245 & 8 & 0.30 & 4.853 & 40 \\
\hline 24 & 107 & 331 & 25 & 132 & 63 & 237 & 7 & 0.45 & 3.729 & 13 \\
\hline 24 & 109 & 330 & 4 & 151 & 85 & 61 & 0 & & & 0 \\
\hline 24 & 111 & 330 & 4 & 151 & 85 & 61 & 0 & & & 0 \\
\hline 24 & 113 & 108 & 13 & 16 & 7 & 258 & 75 & 0.60 & 1.843 & 4 \\
\hline 24 & 115 & 71 & 13 & 310 & 65 & 166 & 20 & 0.50 & 1.226 & 7 \\
\hline 24 & 117 & 292 & 6 & 177 & 75 & 24 & 13 & 0.40 & 10.524 & 210 \\
\hline 24 & 119 & 112 & 3 & 206 & 58 & 20 & 31 & 0.65 & 10.459 & 318 \\
\hline 24 & 121 & 292 & 3 & 189 & 76 & 23 & 13 & 0.40 & 9.989 & 351 \\
\hline 26 & 99 & 190 & 35 & 29 & 53 & 287 & 9 & 0.15 & 11.605 & 99 \\
\hline 26 & 101 & 10 & 35 & 177 & 54 & 276 & 6 & 0.40 & 11.245 & 90 \\
\hline 26 & 103 & 10 & 35 & 163 & 52 & 271 & 13 & 0.25 & 9.879 & 69 \\
\hline 26 & 105 & 158 & 14 & 310 & 74 & 66 & 7 & 0.40 & 7.871 & 41 \\
\hline 26 & 107 & 306 & 10 & 172 & 75 & 38 & 10 & 0.20 & 3.904 & 11 \\
\hline 26 & 111 & 80 & 3 & 175 & 60 & 353 & 29 & & & 0 \\
\hline 26 & 113 & 80 & 3 & 175 & 60 & 353 & 29 & & & 0 \\
\hline 26 & 115 & 75 & 24 & 292 & 60 & 172 & 16 & 0.30 & 2.099 & 8 \\
\hline
\end{tabular}




\begin{tabular}{|c|c|c|c|c|c|c|c|c|c|c|}
\hline \multirow{3}{*}{$\begin{array}{l}\text { Lat. } \\
{ }^{\circ} \mathrm{N}\end{array}$} & \multirow{3}{*}{$\begin{array}{l}\text { Long. } \\
{ }^{\circ} \mathrm{E}\end{array}$} & \multirow{2}{*}{\multicolumn{2}{|c|}{$\sigma_{1}$ axis }} & \multirow{2}{*}{\multicolumn{2}{|c|}{$\sigma_{2}$ axis }} & \multirow{2}{*}{\multicolumn{2}{|c|}{$\sigma_{3}$ axis }} & \multirow{3}{*}{$R$} & \multirow{3}{*}{$\begin{array}{c}\text { Misfit angle } \\
1^{\circ}\end{array}$} & \multirow{3}{*}{$\begin{array}{l}\text { Number of } \\
\text { earthquakes }\end{array}$} \\
\hline & & & & & & & & & & \\
\hline & & $\overline{\mathrm{Az}} /^{\circ}$ & $\mathrm{P} 1 /^{\circ}$ & $\mathrm{Az} /{ }^{\circ}$ & $\mathrm{Pl} /{ }^{\circ}$ & $\overline{\mathrm{Az}} /^{\circ}$ & $\mathrm{Pl} /{ }^{\circ}$ & & & \\
\hline 26 & 117 & 112 & 4 & 221 & 78 & 21 & 11 & 0.45 & 10.029 & 143 \\
\hline 26 & 119 & 99 & 9 & 213 & 68 & 6 & 19 & 0.40 & 10.914 & 246 \\
\hline 28 & 85 & 178 & 14 & 299 & 64 & 83 & 21 & 0.40 & 5.393 & 40 \\
\hline 28 & 87 & 188 & 34 & 343 & 53 & 90 & 12 & 0.40 & 5.471 & 39 \\
\hline 28 & 89 & 188 & 34 & 20 & 55 & 282 & 6 & 0.50 & 7.282 & 58 \\
\hline 28 & 91 & 25 & 14 & 205 & 76 & 295 & 0 & 0.70 & 9.115 & 66 \\
\hline 28 & 93 & 39 & 7 & 144 & 64 & 306 & 24 & 0.95 & 10.821 & 81 \\
\hline 28 & 95 & 39 & 30 & 205 & 59 & 306 & 6 & 0.75 & 10.589 & 85 \\
\hline 28 & 97 & 180 & 50 & 39 & 32 & 296 & 20 & 0.50 & 11.272 & 104 \\
\hline 28 & 99 & 182 & 55 & 40 & 28 & 300 & 18 & 0.50 & 12.105 & 85 \\
\hline 28 & 101 & 10 & 35 & 179 & 54 & 277 & 5 & 0.40 & 12.726 & 79 \\
\hline 28 & 103 & 10 & 35 & 157 & 50 & 268 & 16 & 0.35 & 13.290 & 57 \\
\hline 28 & 105 & 123 & 23 & 321 & 65 & 216 & 7 & 0.70 & 10.506 & 38 \\
\hline 28 & 107 & 293 & 18 & 49 & 53 & 192 & 30 & 0.65 & 2.790 & 10 \\
\hline 28 & 109 & 320 & 5 & 226 & 33 & 58 & 56 & 0.10 & 0.738 & 4 \\
\hline 28 & 111 & 80 & 3 & 175 & 60 & 353 & 29 & & & 0 \\
\hline 28 & 113 & 80 & 3 & 175 & 60 & 353 & 29 & & & 0 \\
\hline 28 & 115 & 69 & 14 & 330 & 29 & 181 & 57 & 0.50 & 0.442 & 4 \\
\hline 28 & 117 & 296 & 3 & 192 & 77 & 27 & 12 & 0.40 & 9.818 & 96 \\
\hline 28 & 119 & 110 & 9 & 211 & 50 & 13 & 38 & 0.65 & 9.432 & 196 \\
\hline 28 & 121 & 110 & 9 & 213 & 55 & 14 & 33 & 0.60 & 9.889 & 220 \\
\hline 30 & 81 & 15 & 5 & 225 & 84 & 105 & 3 & 0.50 & 3.869 & 27 \\
\hline 30 & 83 & 174 & 26 & 317 & 58 & 76 & 16 & 0.20 & 4.795 & 34 \\
\hline 30 & 85 & 186 & 30 & 354 & 59 & 93 & 5 & 0.35 & 5.566 & 60 \\
\hline 30 & 87 & 348 & 61 & 191 & 27 & 96 & 10 & 0.50 & 5.787 & 62 \\
\hline 30 & 89 & 293 & 81 & 23 & 0 & 114 & 9 & 0.85 & 5.429 & 70 \\
\hline 30 & 91 & 269 & 81 & 13 & 2 & 104 & 9 & 0.50 & 6.758 & 78 \\
\hline 30 & 93 & 203 & 66 & 32 & 23 & 301 & 3 & 0.50 & 9.463 & 88 \\
\hline 30 & 95 & 48 & 43 & 200 & 43 & 304 & 14 & 0.65 & 9.660 & 68 \\
\hline 30 & 97 & 240 & 85 & 27 & 4 & 118 & 3 & 0.70 & 9.484 & 81 \\
\hline 30 & 99 & 248 & 55 & 93 & 32 & 356 & 12 & 0.10 & 9.413 & 72 \\
\hline 30 & 101 & 79 & 66 & 291 & 20 & 197 & 12 & 0.60 & 12.469 & 66 \\
\hline 30 & 103 & 293 & 39 & 119 & 50 & 26 & 3 & 0.65 & 9.951 & 48 \\
\hline 30 & 105 & 118 & 32 & 278 & 56 & 22 & 9 & 0.50 & 9.313 & 37 \\
\hline 30 & 107 & 293 & 18 & 49 & 53 & 192 & 30 & 0.65 & 2.769 & 12 \\
\hline 30 & 109 & 272 & 7 & 181 & 0 & 86 & 83 & 0.70 & 1.189 & 5 \\
\hline 30 & 111 & 273 & 1 & 14 & 84 & 187 & 5 & & & 0 \\
\hline 30 & 113 & 77 & 66 & 249 & 23 & 159 & 0 & & & 0 \\
\hline 30 & 115 & 69 & 7 & 233 & 82 & 339 & 2 & & & 0 \\
\hline 30 & 117 & 47 & 14 & 311 & 19 & 170 & 66 & 0.75 & 0.996 & 4 \\
\hline 30 & 119 & 249 & 38 & 96 & 48 & 350 & 14 & 0.40 & 5.530 & 10 \\
\hline 30 & 121 & 282 & 83 & 94 & 6 & 185 & 1 & 0.40 & 5.488 & 21 \\
\hline 32 & 79 & 17 & 7 & 227 & 81 & 108 & 4 & 0.70 & 8.558 & 47 \\
\hline 32 & 81 & 183 & 19 & 338 & 69 & 90 & 8 & 0.20 & 6.646 & 40 \\
\hline 32 & 83 & 178 & 14 & 315 & 71 & 85 & 12 & 0.40 & 6.004 & 47 \\
\hline 32 & 85 & 183 & 23 & 357 & 66 & 92 & 2 & 0.35 & 6.170 & 70 \\
\hline 32 & 87 & 186 & 30 & 341 & 57 & 89 & 11 & 0.40 & 6.194 & 70 \\
\hline 32 & 89 & 200 & 60 & 1 & 28 & 96 & 8 & 0.30 & 7.183 & 84 \\
\hline 32 & 91 & 203 & 66 & 7 & 23 & 100 & 6 & 0.35 & 7.693 & 93 \\
\hline 32 & 93 & 240 & 80 & 30 & 8 & 121 & 5 & 0.60 & 7.675 & 88 \\
\hline 32 & 95 & 45 & 35 & 184 & 47 & 299 & 21 & 0.60 & 9.177 & 67 \\
\hline 32 & 97 & 45 & 35 & 184 & 47 & 299 & 21 & 0.60 & 11.555 & 77 \\
\hline 32 & 99 & 69 & 35 & 245 & 54 & 338 & 2 & 0.30 & 12.522 & 59 \\
\hline 32 & 101 & 69 & 35 & 245 & 54 & 338 & 2 & 0.30 & 10.841 & 52 \\
\hline 32 & 103 & 281 & 9 & 20 & 46 & 183 & 42 & 0.90 & 12.630 & 46 \\
\hline 32 & 105 & 294 & 2 & 26 & 47 & 202 & 42 & 0.50 & 9.529 & 36 \\
\hline 32 & 107 & 297 & 19 & 51 & 49 & 194 & 34 & 0.60 & 3.140 & 12 \\
\hline 32 & 109 & 96 & 0 & 5 & 69 & 186 & 21 & 0.80 & 0.683 & 6 \\
\hline 32 & 111 & 273 & 1 & 14 & 84 & 187 & 5 & & & 0 \\
\hline 32 & 113 & 77 & 66 & 249 & 23 & 159 & 0 & & & 0 \\
\hline 32 & 115 & 252 & 12 & 34 & 74 & 160 & 9 & 0.40 & 0.516 & 5 \\
\hline 32 & 117 & 257 & 7 & 139 & 75 & 349 & 13 & 0.50 & 0.397 & 5 \\
\hline 32 & 119 & 252 & 12 & 34 & 74 & 160 & 9 & 0.40 & 1.065 & 6 \\
\hline
\end{tabular}


Continued from Table 1

\begin{tabular}{|c|c|c|c|c|c|c|c|c|c|c|}
\hline \multirow{2}{*}{$\begin{array}{l}\text { Lat. } \\
{ }^{\circ} \mathrm{N}\end{array}$} & \multirow{2}{*}{$\begin{array}{l}\text { Long. } \\
/{ }^{\circ} \mathrm{E}\end{array}$} & \multicolumn{2}{|c|}{$\sigma_{1}$ axis } & \multicolumn{2}{|c|}{$\sigma_{2}$ axis } & \multicolumn{2}{|c|}{$\sigma_{3}$ axis } & \multirow{2}{*}{$R$} & \multirow{2}{*}{$\begin{array}{l}\text { Misfit angle } \\
/^{\circ}\end{array}$} & \multirow{2}{*}{$\begin{array}{l}\text { Number of } \\
\text { earthquakes }\end{array}$} \\
\hline & & $\mathrm{Az} /{ }^{\circ}$ & $\mathrm{Pl} /^{\circ}$ & $\mathrm{Az} /{ }^{\circ}$ & $\mathrm{Pl} /{ }^{\circ}$ & $\mathrm{Az} /{ }^{\circ}$ & $\mathrm{P1} /{ }^{\circ}$ & & & \\
\hline 32 & 121 & 240 & 5 & 29 & 84 & 150 & 3 & 0.35 & 0.978 & 5 \\
\hline 34 & 79 & 20 & 9 & 212 & 80 & 110 & 2 & 0.60 & 8.484 & 54 \\
\hline 34 & 81 & 18 & 9 & 227 & 79 & 109 & 5 & 0.50 & 7.082 & 43 \\
\hline 34 & 83 & 183 & 19 & 338 & 69 & 90 & 8 & 0.20 & 6.467 & 46 \\
\hline 34 & 85 & 183 & 14 & 3 & 76 & 273 & 0 & 0.35 & 6.261 & 65 \\
\hline 34 & 87 & 0 & 35 & 183 & 54 & 91 & 2 & 0.25 & 6.846 & 72 \\
\hline 34 & 89 & 193 & 19 & 321 & 61 & 96 & 21 & 0.25 & 4.751 & 85 \\
\hline 34 & 91 & 27 & 2 & 275 & 84 & 117 & 5 & 0.50 & 7.279 & 98 \\
\hline 34 & 93 & 29 & 26 & 198 & 63 & 297 & 4 & 0.50 & 8.299 & 100 \\
\hline 34 & 95 & 26 & 23 & 206 & 67 & 116 & 0 & 0.50 & 8.669 & 78 \\
\hline 34 & 97 & 52 & 7 & 149 & 45 & 315 & 44 & 0.65 & 9.842 & 76 \\
\hline 34 & 99 & 57 & 7 & 153 & 44 & 320 & 45 & 0.65 & 8.992 & 59 \\
\hline 34 & 101 & 217 & 14 & 118 & 31 & 328 & 55 & 0.65 & 11.898 & 51 \\
\hline 34 & 103 & 77 & 25 & 241 & 64 & 344 & 6 & 0.15 & 8.313 & 40 \\
\hline 34 & 105 & 112 & 0 & 22 & 47 & 202 & 43 & 0.50 & 6.978 & 31 \\
\hline 34 & 107 & 98 & 0 & 8 & 51 & 188 & 39 & 0.85 & 5.171 & 14 \\
\hline 34 & 109 & 84 & 30 & 255 & 59 & 352 & 4 & 0.50 & 2.363 & 8 \\
\hline 34 & 111 & 82 & 6 & 347 & 35 & 180 & 54 & 0.55 & 1.368 & 5 \\
\hline 34 & 113 & 256 & 37 & 39 & 46 & 151 & 19 & 0.45 & 0.943 & 4 \\
\hline 34 & 115 & 90 & 5 & 354 & 45 & 185 & 44 & 0.35 & 1.711 & 6 \\
\hline 34 & 117 & 82 & 22 & 332 & 39 & 194 & 42 & 0.20 & 1.592 & 6 \\
\hline 34 & 119 & 236 & 28 & 53 & 61 & 145 & 1 & 0.20 & 2.530 & 7 \\
\hline 36 & 77 & 202 & 26 & 316 & 40 & 89 & 38 & 0.40 & 10.604 & 121 \\
\hline 36 & 79 & 16 & 21 & 175 & 67 & 283 & 7 & 0.50 & 9.711 & 85 \\
\hline 36 & 81 & 8 & 13 & 192 & 76 & 98 & 1 & 0.25 & 7.084 & 61 \\
\hline 36 & 83 & 357 & 18 & 131 & 64 & 262 & 17 & 0.50 & 6.682 & 33 \\
\hline 36 & 85 & 192 & 7 & 300 & 68 & 99 & 20 & 0.25 & 4.860 & 40 \\
\hline 36 & 87 & 26 & 0 & 296 & 74 & 116 & 16 & 0.50 & 3.971 & 50 \\
\hline 36 & 89 & 21 & 0 & 291 & 77 & 111 & 13 & 0.40 & 6.058 & 67 \\
\hline 36 & 91 & 210 & 5 & 320 & 76 & 119 & 13 & 0.55 & 6.530 & 75 \\
\hline 36 & 93 & 30 & 35 & 190 & 53 & 293 & 9 & 0.45 & 7.097 & 81 \\
\hline 36 & 95 & 30 & 0 & 120 & 46 & 300 & 44 & 0.65 & 8.440 & 63 \\
\hline 36 & 97 & 210 & 9 & 101 & 64 & 305 & 24 & 0.60 & 8.492 & 60 \\
\hline 36 & 99 & 233 & 2 & 142 & 10 & 333 & 79 & 0.35 & 5.430 & 42 \\
\hline 36 & 101 & 57 & 6 & 323 & 33 & 156 & 56 & 0.45 & 5.642 & 39 \\
\hline 36 & 103 & 35 & 0 & 125 & 20 & 305 & 70 & 0.40 & 6.306 & 27 \\
\hline 36 & 105 & 103 & 5 & 9 & 33 & 201 & 56 & 0.55 & 6.761 & 27 \\
\hline 36 & 107 & 101 & 0 & 11 & 42 & 191 & 48 & 0.80 & 5.843 & 15 \\
\hline 36 & 109 & 81 & 26 & 256 & 63 & 351 & 2 & 0.45 & 4.261 & 15 \\
\hline 36 & 111 & 73 & 7 & 333 & 53 & 168 & 36 & 0.65 & 3.689 & 11 \\
\hline 36 & 113 & 258 & 7 & 356 & 49 & 162 & 40 & 0.60 & 4.463 & 13 \\
\hline 36 & 115 & 71 & 0 & 341 & 69 & 161 & 21 & 0.50 & 3.578 & 16 \\
\hline 36 & 117 & 256 & 5 & 356 & 64 & 164 & 25 & 0.55 & 3.566 & 16 \\
\hline 36 & 119 & 252 & 9 & 12 & 72 & 160 & 15 & 0.50 & 3.468 & 14 \\
\hline 38 & 75 & 182 & 7 & 275 & 25 & 78 & 63 & 0.50 & 10.313 & 161 \\
\hline 38 & 77 & 201 & 21 & 309 & 39 & 89 & 43 & 0.40 & 9.903 & 140 \\
\hline 38 & 79 & 22 & 19 & 144 & 57 & 283 & 26 & 0.75 & 9.244 & 102 \\
\hline 38 & 81 & 8 & 13 & 179 & 76 & 278 & 2 & 0.25 & 9.281 & 77 \\
\hline 38 & 83 & 13 & 7 & 193 & 83 & 283 & 0 & 0.30 & 9.885 & 43 \\
\hline 38 & 85 & 194 & 16 & 316 & 62 & 97 & 22 & 0.25 & 6.193 & 49 \\
\hline 38 & 87 & 195 & 14 & 314 & 63 & 99 & 22 & 0.25 & 6.082 & 52 \\
\hline 38 & 89 & 31 & 21 & 217 & 68 & 122 & 2 & 0.55 & 6.331 & 55 \\
\hline 38 & 91 & 216 & 21 & 13 & 67 & 123 & 8 & 0.50 & 7.551 & 62 \\
\hline 38 & 93 & 207 & 3 & 313 & 79 & 116 & 10 & 0.50 & 7.289 & 67 \\
\hline 38 & 95 & 25 & 1 & 283 & 84 & 115 & 5 & 0.50 & 8.155 & 47 \\
\hline 38 & 97 & 25 & 25 & 159 & 56 & 285 & 21 & 0.65 & 4.746 & 44 \\
\hline 38 & 99 & 55 & 0 & 145 & 7 & 325 & 83 & 0.45 & 6.669 & 39 \\
\hline 38 & 101 & 10 & 4 & 279 & 5 & 136 & 83 & 0.50 & 5.326 & 34 \\
\hline 38 & 103 & 227 & 7 & 134 & 21 & 334 & 67 & 0.55 & 6.422 & 24 \\
\hline 38 & 105 & 48 & 27 & 307 & 19 & 186 & 55 & 0.45 & 7.145 & 24 \\
\hline 38 & 107 & 52 & 26 & 312 & 19 & 190 & 57 & 0.05 & 4.428 & 13 \\
\hline 38 & 109 & 36 & 30 & 286 & 30 & 160 & 45 & 0.75 & 7.116 & 13 \\
\hline 38 & 111 & 252 & 5 & 144 & 74 & 343 & 15 & 0.50 & 3.993 & 14 \\
\hline
\end{tabular}




\begin{tabular}{|c|c|c|c|c|c|c|c|c|c|c|}
\hline \multirow{2}{*}{$\begin{array}{l}\text { Lat. } \\
{ }^{\circ} \mathrm{N}\end{array}$} & \multirow{2}{*}{$\begin{array}{l}\text { Long. } \\
{ }^{\circ} \mathrm{E}\end{array}$} & \multicolumn{2}{|c|}{$\sigma_{1}$ axis } & \multicolumn{2}{|c|}{$\sigma_{2}$ axis } & \multicolumn{2}{|c|}{$\sigma_{3}$ axis } & \multirow{2}{*}{$R$} & \multirow{2}{*}{$\begin{array}{l}\text { Misfit angle } \\
/^{\circ}\end{array}$} & \multirow{2}{*}{$\begin{array}{l}\text { Number of } \\
\text { earthquakes }\end{array}$} \\
\hline & & $\mathrm{Az} /{ }^{\circ}$ & $\mathrm{Pl} /^{\circ}$ & $\mathrm{Az} /{ }^{\circ}$ & $\mathrm{Pl} /{ }^{\circ}$ & $\mathrm{Az} / /^{\circ}$ & $\mathrm{Pl} /^{\circ}$ & & & \\
\hline 38 & 113 & 77 & 0 & 167 & 65 & 347 & 25 & 0.45 & 5.677 & 15 \\
\hline 38 & 115 & 255 & 4 & 353 & 65 & 163 & 24 & 0.55 & 3.829 & 17 \\
\hline 38 & 117 & 255 & 4 & 353 & 65 & 163 & 24 & 0.55 & 3.552 & 16 \\
\hline 38 & 119 & 271 & 26 & 36 & 50 & 166 & 28 & 0.75 & 3.644 & 13 \\
\hline 38 & 121 & 257 & 18 & 46 & 69 & 164 & 10 & 0.50 & 3.324 & 11 \\
\hline 38 & 123 & 255 & 9 & 117 & 77 & 346 & 8 & 0.40 & 3.632 & 9 \\
\hline 40 & 75 & 187 & 18 & 96 & 2 & 357 & 72 & 0.40 & 9.082 & 143 \\
\hline 40 & 77 & 22 & 14 & 153 & 69 & 288 & 15 & 0.45 & 9.387 & 123 \\
\hline 40 & 79 & 196 & 26 & 307 & 36 & 80 & 42 & 0.55 & 9.377 & 101 \\
\hline 40 & 81 & 10 & 5 & 220 & 84 & 100 & 3 & 0.20 & 9.639 & 81 \\
\hline 40 & 83 & 208 & 14 & 112 & 22 & 328 & 63 & 0.15 & 9.015 & 47 \\
\hline 40 & 85 & 198 & 14 & 308 & 54 & 99 & 32 & 0.20 & 8.710 & 34 \\
\hline 40 & 87 & 193 & 14 & 309 & 60 & 96 & 25 & 0.30 & 8.294 & 32 \\
\hline 40 & 89 & 210 & 9 & 335 & 74 & 118 & 12 & 0.50 & 8.104 & 35 \\
\hline 40 & 91 & 44 & 0 & 134 & 34 & 314 & 56 & 0.40 & 8.196 & 38 \\
\hline 40 & 93 & 41 & 0 & 131 & 30 & 311 & 60 & 0.40 & 7.477 & 40 \\
\hline 40 & 95 & 41 & 0 & 131 & 30 & 311 & 60 & 0.40 & 6.624 & 37 \\
\hline 40 & 97 & 198 & 9 & 359 & 80 & 108 & 3 & 0.15 & 7.043 & 40 \\
\hline 40 & 99 & 217 & 7 & 307 & 3 & 67 & 82 & 0.30 & 5.407 & 35 \\
\hline 40 & 101 & 198 & 21 & 45 & 66 & 292 & 10 & 0.40 & 6.529 & 30 \\
\hline 40 & 103 & 207 & 14 & 71 & 70 & 300 & 13 & 0.50 & 5.995 & 22 \\
\hline 40 & 105 & 28 & 31 & 269 & 38 & 144 & 36 & 0.70 & 7.142 & 23 \\
\hline 40 & 107 & 40 & 24 & 192 & 63 & 305 & 11 & 0.10 & 7.854 & 12 \\
\hline 40 & 109 & 69 & 36 & 250 & 53 & 159 & 1 & 0.25 & 5.081 & 13 \\
\hline 40 & 111 & 83 & 4 & 182 & 66 & 351 & 23 & 0.60 & 3.604 & 16 \\
\hline 40 & 113 & 73 & 7 & 315 & 75 & 165 & 13 & 0.55 & 2.357 & 17 \\
\hline 40 & 115 & 255 & 4 & 352 & 60 & 163 & 29 & 0.50 & 3.901 & 18 \\
\hline 40 & 117 & 258 & 18 & 144 & 51 & 0 & 33 & 0.30 & 3.033 & 17 \\
\hline 40 & 119 & 87 & 14 & 206 & 63 & 351 & 22 & 0.35 & 2.839 & 14 \\
\hline 40 & 121 & 87 & 19 & 214 & 60 & 349 & 22 & 0.25 & 2.627 & 11 \\
\hline 40 & 123 & 87 & 19 & 214 & 60 & 349 & 22 & 0.25 & 1.046 & 8 \\
\hline 42 & 81 & 351 & 0 & 261 & 4 & 81 & 86 & 0.35 & 7.101 & 70 \\
\hline 42 & 83 & 7 & 13 & 99 & 9 & 226 & 74 & 0.50 & 3.737 & 35 \\
\hline 42 & 85 & 2 & 7 & 93 & 13 & 245 & 75 & 0.65 & 3.945 & 24 \\
\hline 42 & 87 & 2 & 7 & 94 & 22 & 256 & 66 & 0.65 & 4.624 & 23 \\
\hline 42 & 89 & 12 & 2 & 102 & 9 & 271 & 80 & 0.70 & 3.962 & 22 \\
\hline 42 & 91 & 12 & 2 & 102 & 9 & 270 & 80 & 0.70 & 5.813 & 23 \\
\hline 42 & 93 & 45 & 9 & 312 & 17 & 161 & 70 & 0.25 & 5.910 & 30 \\
\hline 42 & 95 & 23 & 14 & 118 & 22 & 263 & 63 & 0.55 & 6.033 & 26 \\
\hline 42 & 97 & 23 & 14 & 117 & 17 & 257 & 67 & 0.55 & 4.924 & 23 \\
\hline 42 & 99 & 200 & 5 & 290 & 7 & 76 & 81 & 0.35 & 5.263 & 23 \\
\hline 42 & 101 & 28 & 14 & 238 & 73 & 120 & 8 & 0.25 & 5.408 & 19 \\
\hline 42 & 103 & 34 & 31 & 231 & 57 & 129 & 8 & 0.75 & 3.762 & 11 \\
\hline 42 & 105 & 66 & 31 & 228 & 57 & 331 & 8 & 0.25 & 5.414 & 14 \\
\hline 42 & 107 & 85 & 0 & 175 & 75 & 355 & 15 & 0.80 & 5.493 & 11 \\
\hline 42 & 109 & 87 & 9 & 197 & 66 & 353 & 22 & 0.65 & 4.535 & 13 \\
\hline 42 & 111 & 83 & 7 & 191 & 68 & 350 & 20 & 0.60 & 3.553 & 15 \\
\hline 42 & 113 & 243 & 19 & 79 & 70 & 335 & 5 & 0.35 & 4.994 & 15 \\
\hline 42 & 115 & 252 & 2 & 344 & 53 & 161 & 36 & 0.45 & 2.829 & 17 \\
\hline 42 & 117 & 87 & 14 & 222 & 70 & 354 & 13 & 0.45 & 4.328 & 17 \\
\hline 42 & 119 & 252 & 2 & 344 & 53 & 161 & 36 & 0.45 & 3.759 & 14 \\
\hline 42 & 121 & 252 & 2 & 345 & 58 & 161 & 31 & 0.40 & 3.242 & 12 \\
\hline 42 & 123 & 252 & 7 & 353 & 58 & 158 & 31 & 0.40 & 2.048 & 9 \\
\hline 42 & 125 & 62 & 14 & 275 & 73 & 149 & 10 & & & 0 \\
\hline 42 & 127 & 62 & 14 & 275 & 73 & 149 & 10 & & & 0 \\
\hline 44 & 81 & 186 & 18 & 290 & 37 & 76 & 47 & 0.50 & 7.928 & 67 \\
\hline 44 & 83 & 358 & 9 & 88 & 4 & 203 & 80 & 0.55 & 5.929 & 33 \\
\hline 44 & 85 & 8 & 14 & 100 & 9 & 222 & 73 & 0.50 & 3.994 & 28 \\
\hline 44 & 87 & 192 & 7 & 284 & 18 & 82 & 70 & 0.60 & 3.776 & 23 \\
\hline 44 & 89 & 192 & 7 & 284 & 18 & 82 & 70 & 0.70 & 4.057 & 20 \\
\hline 44 & 91 & 192 & 2 & 282 & 17 & 96 & 72 & 0.65 & 3.616 & 16 \\
\hline 44 & 93 & 196 & 17 & 83 & 50 & 298 & 34 & 0.65 & 6.306 & 17 \\
\hline 44 & 95 & 14 & 0 & 284 & 21 & 104 & 68 & 0.65 & 3.896 & 11 \\
\hline
\end{tabular}




\begin{tabular}{|c|c|c|c|c|c|c|c|c|c|c|}
\hline & & & & & & & & & Cont & 1 from Table 1 \\
\hline \multirow{2}{*}{$\begin{array}{l}\text { Lat. } \\
/{ }^{\circ} \mathrm{N}\end{array}$} & \multirow{2}{*}{$\begin{array}{l}\text { Long. } \\
/{ }^{\circ} \mathrm{E}\end{array}$} & \multicolumn{2}{|c|}{$\sigma_{1}$ axis } & \multicolumn{2}{|c|}{$\sigma_{2}$ axis } & \multicolumn{2}{|c|}{$\sigma_{3}$ axis } & \multirow{2}{*}{$R$} & \multirow{2}{*}{$\begin{array}{l}\text { Misfit angle } \\
/{ }^{\circ}\end{array}$} & \multirow{2}{*}{$\begin{array}{l}\text { Number of } \\
\text { earthquakes }\end{array}$} \\
\hline & & $\mathrm{Az} /^{\circ}$ & $\mathrm{Pl} /{ }^{\circ}$ & $\mathrm{Az} /^{\circ}$ & $\mathrm{Pl} /{ }^{\circ}$ & $\mathrm{Az} /^{\circ}$ & $\mathrm{Pl} /^{\circ}$ & & & \\
\hline 44 & 97 & 21 & 21 & 228 & 66 & 115 & 10 & 0.35 & 2.494 & 9 \\
\hline 44 & 113 & 68 & 23 & 250 & 66 & 158 & 1 & 0.30 & 4.537 & 14 \\
\hline 44 & 115 & 253 & 7 & 353 & 56 & 158 & 33 & 0.50 & 4.105 & 16 \\
\hline 44 & 117 & 87 & 14 & 222 & 70 & 354 & 13 & 0.45 & 4.202 & 17 \\
\hline 44 & 119 & 69 & 0 & 339 & 56 & 159 & 34 & 0.40 & 2.537 & 14 \\
\hline 44 & 121 & 81 & 5 & 184 & 69 & 349 & 20 & 0.25 & 3.694 & 12 \\
\hline 44 & 123 & 90 & 14 & 210 & 63 & 355 & 22 & 0.35 & 2.895 & 9 \\
\hline 44 & 125 & 259 & 23 & 77 & 66 & 172 & 2 & & & 0 \\
\hline 44 & 127 & 251 & 14 & 102 & 73 & 336 & 10 & & & 0 \\
\hline 44 & 129 & 251 & 14 & 102 & 73 & 336 & 10 & & & 0 \\
\hline 44 & 131 & 251 & 14 & 102 & 73 & 336 & 10 & & & 0 \\
\hline 44 & 133 & 251 & 14 & 102 & 73 & 336 & 10 & & & 0 \\
\hline 46 & 83 & 354 & 0 & 84 & 4 & 264 & 86 & 0.65 & 6.433 & 36 \\
\hline 46 & 85 & 9 & 5 & 100 & 19 & 265 & 70 & 0.65 & 5.398 & 41 \\
\hline 46 & 87 & 189 & 5 & 280 & 11 & 76 & 77 & 0.70 & 6.632 & 37 \\
\hline 46 & 89 & 180 & 5 & 271 & 15 & 72 & 74 & 0.70 & 5.905 & 34 \\
\hline 46 & 91 & 0 & 0 & 90 & 43 & 270 & 47 & 0.60 & 4.864 & 30 \\
\hline 46 & 117 & 278 & 18 & 179 & 25 & 39 & 58 & 0.05 & 1.115 & 6 \\
\hline 46 & 119 & 278 & 18 & 179 & 25 & 39 & 58 & 0.05 & 0.978 & 6 \\
\hline 46 & 121 & 288 & 12 & 31 & 48 & 188 & 39 & 0.75 & 0.735 & 4 \\
\hline 46 & 123 & 259 & 23 & 77 & 66 & 172 & 2 & & & 0 \\
\hline 46 & 125 & 251 & 14 & 102 & 73 & 336 & 10 & & & 0 \\
\hline 46 & 127 & 251 & 14 & 102 & 73 & 336 & 10 & & & 0 \\
\hline 46 & 129 & 251 & 14 & 102 & 73 & 336 & 10 & & & 0 \\
\hline 46 & 131 & 251 & 14 & 102 & 73 & 336 & 10 & & & 0 \\
\hline 46 & 133 & 251 & 14 & 102 & 73 & 336 & 10 & & & 0 \\
\hline 48 & 81 & 347 & 7 & 250 & 41 & 85 & 48 & 0.50 & 3.889 & 13 \\
\hline 48 & 87 & 355 & 0 & 265 & 20 & 85 & 70 & 0.65 & 6.147 & 31 \\
\hline 48 & 117 & 288 & 12 & 31 & 48 & 188 & 39 & 0.75 & 0.735 & 4 \\
\hline 48 & 119 & 277 & 18 & 177 & 26 & 37 & 57 & 0.05 & 0.481 & 4 \\
\hline 48 & 121 & 90 & 9 & 199 & 64 & 356 & 24 & 0.50 & 0.842 & 4 \\
\hline 48 & 123 & 259 & 23 & 77 & 66 & 172 & 2 & & & 0 \\
\hline 48 & 125 & 259 & 23 & 77 & 66 & 172 & 2 & & & 0 \\
\hline 48 & 127 & 251 & 14 & 102 & 73 & 336 & 10 & & & 0 \\
\hline 48 & 129 & 251 & 14 & 102 & 73 & 336 & 10 & & & 0 \\
\hline 48 & 131 & 251 & 14 & 102 & 73 & 336 & 10 & & & 0 \\
\hline 50 & 121 & 259 & 23 & 77 & 66 & 172 & 2 & & & 0 \\
\hline 50 & 123 & 259 & 23 & 77 & 66 & 172 & 2 & & & 0 \\
\hline 50 & 125 & 259 & 23 & 77 & 66 & 172 & 2 & & & 0 \\
\hline 50 & 127 & 251 & 14 & 102 & 73 & 336 & 10 & & & 0 \\
\hline 52 & 119 & 58 & 14 & 176 & 62 & 322 & 23 & 0.50 & 2.238 & 5 \\
\hline 52 & 121 & 60 & 35 & 213 & 52 & 321 & 13 & 0.20 & 0.688 & 4 \\
\hline
\end{tabular}

Note: The data with Number of earthquakes of 0 are from Wang and Xu (1985).

Most of $\sigma_{1}$ and $\sigma_{3}$ axes are horizontal (Figures 1 and 2), indicating that the seismotectonic deformation takes place primarily through strike-slip faulting. The continuous $\sigma_{1}$ and $\sigma_{3}$ direction showing the stress field in China and its adjacent areas has the same source which is driven by the northward indentation of the Indian plate, and subduction of the Pacific ocean plate under the Eurasia plate. The western part, i.e., Tibetan plateau, undergoes north-south compression and east-west extension, and this trend extends to Xinjiang area. The $\sigma_{1}$ direction appears to curve sharply in the eastern end of Himalaya arc, showing some eddylike feature to the south of the Assam wedge (Figure 1). The principal stress axes show a relatively uniform radial pattern. That is, the compressive horizontal stress trajectories radiate from Tibet plateau to the northern, eastern, and southeastern parts of the mainland (Figure 1). The inferred extensional stress directions lie along arcs convex outward from the plateau (Figure 2). Despite inevitable local variations, which are often indistinguishable from the error in stress axis estimation, this overall pattern is schematically expressed in Figure 1 by horizontal stress trajectories.

In central part of Tibet plateau, there are relative vertical $\sigma_{1}$ axes, horizontal $\sigma_{3}$ axes in east-west direction. This may be caused by the extension of this region and 


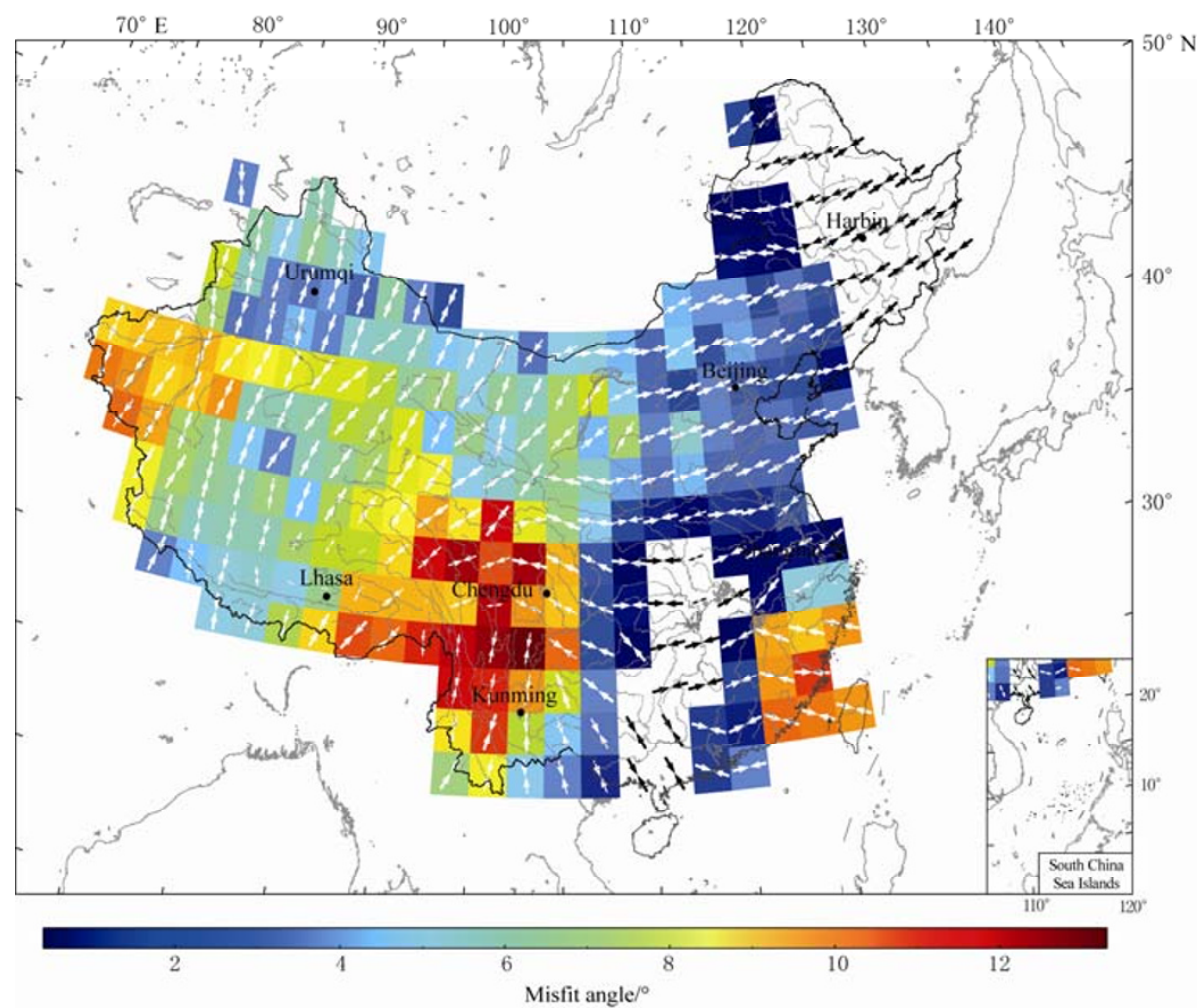

Figure $1 \sigma_{1}$ direction and misfit angles obtained in this study. Quivers show the directions of $\sigma_{1}$, and the longer the quiver is, the more horizontal the $\sigma_{1}$ will be.

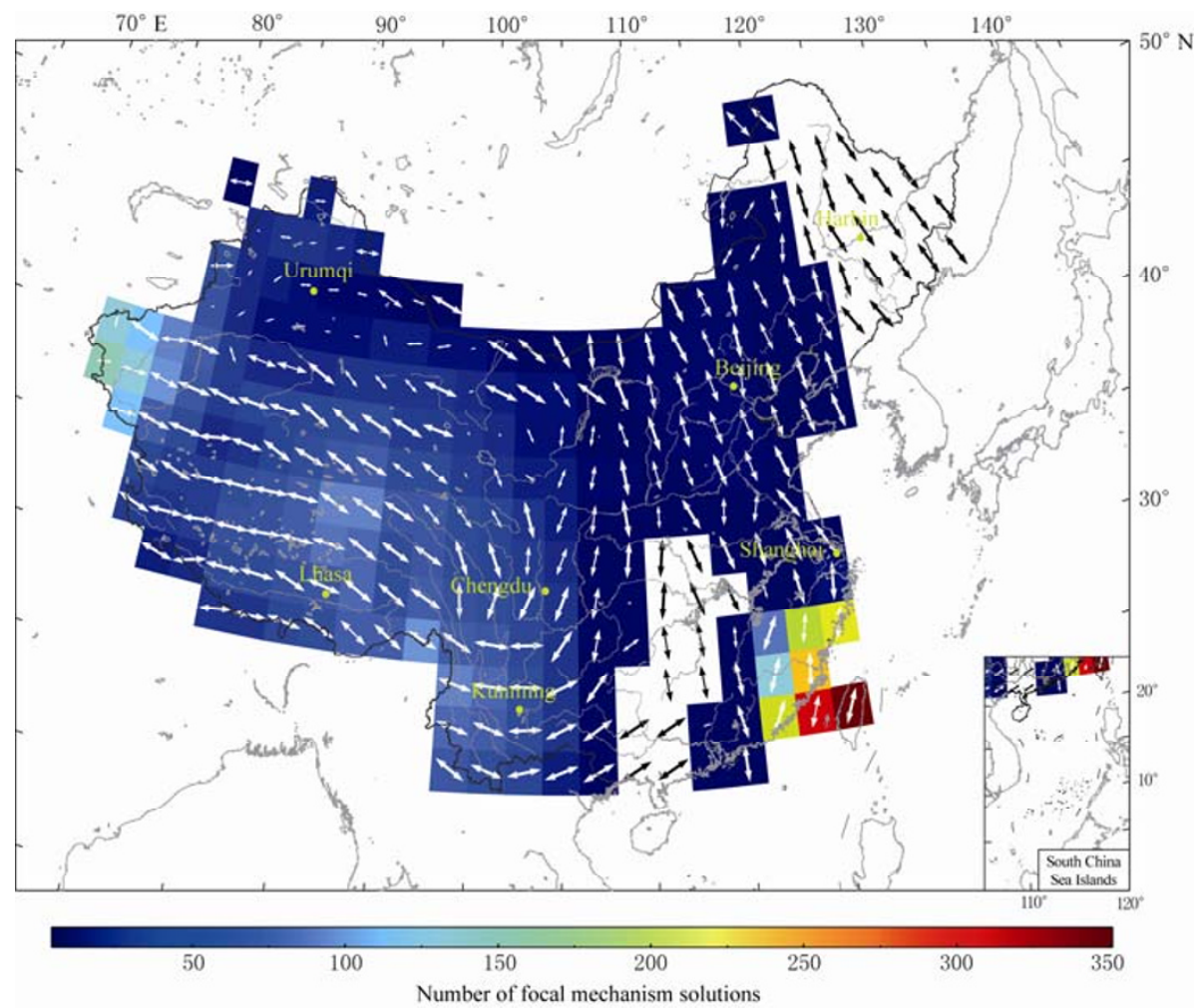

Figure $2 \sigma_{3}$ direction and number of data used in this study. Quivers show the directions of $\sigma_{3}$, and the longer the quiver is, the more horizontal the $\sigma_{3}$ will be. 
be validated by more lakes in this area. In the North-South Seismic Zones, we can see that the stress direction changes sharply, which indicates a dividing line of the stress field in this area.

\section{Discussion and conclusions}

The data used to infer modern stress field in the present study come from earthquakes occurred during the past several decades, stress measurements in recent years and quaternary fault slip measurements. It is interesting to notice that a similar pattern of stress axes as discovered in this study has also been found before. For example, Xu et al (1992) used a different method to get the mean principal tress axes basing on $9621 \mathrm{P}$ wave first motion polarity from 5054 small earthquakes. Using the similar method to this study, Du and Shao (1999) also got the modern tectonic stress field. Based on the data of earthquake centroid moment tensor (CMT) solution, P-wave first motion focal mechanism solution and deep hole breakouts, $\mathrm{Xu}$ (2001) compiled a present-day tectonic stress map for eastern Asia region. The same stress field pattern confirms our study method and data used. But the stress filed inferred in this study covers more areas. The stress field obtained in this study is also consistent with GPS measurement (Wang et al, 2001; Wang et al, 2003) and its strain rate field (Shen et al, 2003; Zhang et al, 2004), quaternary fault slip rates and GPS observations (Holt et al, 2000), as well as GPS, geologic, and shear wave splitting data (Flesh et al, 2005).

Existence of the broad-scale radial pattern of $\sigma_{1}$ directions indicates that the primary force responsible for the tectonic movement and earthquake generation in continental area of China does not come from some local sources but from an external driving force on a large scale. It is quite likely related to the indentation effect of plate collision between India and Euroasia, as studied by many authors (e.g., Tapponnier and Molnar, 1976; Houseman and England, 1986; England and Houseman, 1986).

As mentioned previously, we have studied a stress field in some crustal volume which has a thickness represented by earthquake focal depth. Due to the limited resolving capability of the method and data that we used, we can say nothing about possible variation of the stress state with depth.

Most of stress relief measurement data are measured in shallow part of the crust. Strictly speaking, they cannot be used to invert the stress field in the deep part of the crust (e.g., $\mathrm{Xu}, 2001$ ) for being affected by topography. In this study, we want to get the average stress field in a relatively large area, the topographic effect may be smoothed, so this sort of data can be used to constrain the stress field.

In the stress filed inversion, we select the data within the square areas of $5^{\circ} \times 5^{\circ}$ with the center of grid point. So the smoothing stress field can be achieved, and can be conveniently used to constrain the stress field in geodynamic process simulation, earthquake prediction (e.g., Peng et al, 2000; Wan, 2004) as well as slip property of active fault determination (Wan et al, 2008).

Acknowledgements This work is supported by the National Natural Science Foundation of China (40874022), Public Utility Research Project (200808053) and 973 program (2008CB425703). We would like to thank John Gephart for making his program available and Furen Xie for making Chinese stress field data available. Profs. Zhonghuai Xu and Zhengkang Shen provided their constructive comments and suggestions. The reviewers' comments improved the manuscript a lot.

\section{References}

Aki K (1966). Earthquake generating stress in Japan for the years 1961 to 1963 obtained by smoothing the first motion patterns. Bull Earthq Res Inst 44: $447-471$.

Angelier J (1979). Determination of the mean principal direction of stress for a given population. Tectonophysics 56: T17-T26.

Angelier J (1989). From orientation to magnitudes in paleostress determination using fault slip data. $J$ Struct Geol 11: 37-50.

Cui X F and Xie F R (1999). Preliminary research to determine stress districts from focal mechanism solutions in Southwest China and its adjacent area. Acta Seismologica Sinica 12(5): 562-572.

Du X X and Shao H C (1999). Modern tectonic stress field in the Chinese mainland inverted from focal mechanism solutions. Acta Seismologica Sinica 12(4): 390-397.

England P C and Houseman G A (1986). Finite strain calculations of continental deformation 2, comparison with the India-Asia collision zone. $J$ Geophys Res 91: 3 664-3 676.

Flesch L M, Holt W E, Silver P G, Stephenson M, Wang C Y and Chan W W (2005). Constraining the extent of crust-mantle coupling in central Asia using GPS, geologic, and shear wave splitting data. Earth Planet Sci Lett 238: $248-268$

Gephart J W (1990). FMSI: A fortran program for inverting fault/slickenside and earthquake focal mechanism data to obtain the original stress tensor. Comput Geosci 16: 953-989.

Gephart J W and Forsyth D W (1984). An improved method for determining the regional stress tensor using focal mechanism data: application to the San Fernando earthquake sequence. J Geophys Res 89(B11): 9 305-9 320.

Gillard D, Wyss M and Okubo P (1996). Type of faulting and orientation of stress and strain as a function of space and time in Kilauea's south flank, Hawaii. J Geophys Res 101: 16 025-16 042.

Hardebeck J L and Hauksson E (2001). Stress orientations obtained from earthquake focal mechanisms: what are appropriate uncertainty estimates? Bull Seism Soc Amer 91: 250-262.

Holt W E, Chamot-Rooke N, Le Pichon X, Haines A J, Shen-Tu B and Ren J (2000). Velocity field in Asia inferred from Quaternary fault slip rates and global positioning system observations. J Geophys Res 105: 19 185-19 209. 
Horiuchi S, Russo G and Hasegawa A (1995). Discrimination of fault planes from auxiliary planes based on simultaneous determination of stress tensor and a large number of fault plane solutions. J Geophys Res 100: 8 3278338 .

Houseman G A and England P C (1986). Finite strain calculations of continental deformation 1 , method and general results for convergent zones. $J$ Geophys Res 91: 3 651-3 663.

Kang Y, Yang X, Chen X, Chen G M and Zheng S H (2008). Inversion of stress field in Guangdong and its adjacent area. Acta Seismologica Sinica 21(1): 58-66.

Li Q Z, Wang Z G, Chia Y N and Chin Y M (1973). Stress field obtained for two regions from weak earthquake data recorded at a single station. Chinese J Geophys 16: 49-61 (in Chinese with English abstract).

$\mathrm{Lu} \mathrm{Z}$ and Wyss M (1996). Segmentation of the Aleutian plate boundary derived from stress direction estimates based on fault plane solutions. $J$ Geophy Res 101: 803-816.

Lu Z, Wyss M and Pulpan H (1997). Details of stress directions in the Alaska subduction zone from fault plane solutions. J Geophy Res 102: 5 3855402.

Michael A J (1984). Determination of stress from slip data: faults and folds. $J$ Geophy Res 89: 11 517-11 526.

Michael A J (1987). Use of focal mechanisms to determine stress: a control study. J Geophys Res 92: 357-368.

Peng K Y, Yin X C, Wang H T and Zhang Y X (2000). Study on loading/unloading response ratio based on the tectonic stress field. Earthquake Research in China 16(2): 190-196 (in Chinese with English abstract).

Shen Z K, Wang M, Gan W J and Zhang Z S (2003). Contemporary tectonic strain rate field of Chinese continent and its geodynamic implications. Earth Science Frontiers 10(Suppl): 93-100 (in Chinese with English abstract).

Tapponnier P and Molnar P (1976). Slip-line field theory and large scale continental tectonics. Nature 294: 319-324.

Wan Y G (2004). Some considerations on theory of load and unload response ratio and its application to earthquake prediction. Northwestern Seismological Journal 26(2): 178-181 (in Chinese with English abstract).

Wan Y G, Shen Z K, Diao G L, Wang F C, Hu X L and Sheng S Z (2008). An algorithm of fault parameter determination using distribution of small earthquakes and parameters of regional stress field and its application to Tangshan earthquake sequence. Chinese J Geophys 51(3): 569-583.

Wang M, Shen Z K, Niu Z J, Sun H R, Gan W J, Wang Q and Ren Q (2003). Contemporary crustal deformation of the Chinese continent and tectonic block model. Science in China (Series D) 33(Suppl): 21-32 (in Chinese).

Wang Q, Zhang P Z, Freymueller J, Bilham R, Larson K, Lai X, You X, Niu Z, Wu J, Li Y, Liu J, Yang Z and Chen Q (2001). Presentday crustal deformation in China constrained by Global Positioning System (GPS) measurements. Science 294: 574-577.
Wang S Y and Xu Z H (1985). Seismo-tectonic stress field in east China. Acta Seismologica Sinica 7(1): 17-32 (in Chinese with English abstract).

Wyss M, Liang B, Tanigawa W R and Wu X (1992). Comparison of orientations of stress and strain tensors based on fault plane solutions in Kaoiki Hawaii. J Geophys Res 97: 4 769-4 790.

Wyss M and Lu Z (1995). Plate boundary segmentation by stress directions: southern San Andreas fault, California. Geophys Res Lett 22: 547-550.

Xie F R, Chen Q C, Cui X F, Li H, Yang S X, Guo Q L, Chen L W, Xu Z H, Zhang Y S, Dou S Q, Zhao J T, Zhang Z S, Liu C Y and Wang G J (2003). Database of crustal stress in China and its adjacent areas. In: Chen Q C, Cui X F, Li H, Yang S X and Chen L W eds. Crustal Stress in China. Geological Press, Beijing, 3-12 (in Chinese with English abstract).

Xie F R, Cui X F, Zhao J T, Chen Q C and Li H (2004). Regional division of the recent tectonic stress field in China and adjacent areas. Chinese $J$ Geophys 47(4): 654-662 (in Chinese with English abstract).

Xie F R, Zhu J Z, Liang H Q and Liu G X (1993). Basic characteristics of recent tectonic stress field in southwest China. Acta Seismologica Sinica 6(4): 843-855.

$\mathrm{Xu}$ Z H (2001). A present-day tectonic stress map for Eastern Asia region. Acta Seismologica Sinica 14(5): 524-533.

Xu Z H and Ge S M (1984). Stress field in the Fuyun, Xinjiang earthquake fracture zone determined by fitting fault slip vector data. Acta Seismologica Sinica 6(4): 395-404 (in Chinese with English abstract).

Xu Z H, Wang S Y, Huang Y R and Gao A J (1992). Tectonic stress field of China inferred from a large number of small earthquakes. $J$ Geophys Res 97: $11867-11877$.

Xu Z H, Wang S Y, Huang Y R, Gao A J, Jin X F and Chang X D (1987). Directions of mean stress axes in southwestern China deduced from microearthquake data. Chinese J Geophys 30(5): 476-486 (in Chinese).

Xu Z H, Yan M and Zhao Z H (1983). Evaluation of direction of tectonic stress in North China from recorded data of a large number of small earthquakes. Acta Seismologica Sinica 5(3): 268-279.

Xue H Y and Yan J Q (1984). The contemporary stress field around the Ordos block. Chinese J Geophys 27(2): 144-152 (in Chinese with English abstract).

Zhang P Z, Shen Z K, Wang M, Gan W J, Buergmann R, Molnar P, Wang Q, Niu Z J, Sun J Z, Wu J C, Sun H R and You X Z (2004). Continuous deformation of the Tibetan Plateau from global positioning system data. Geology 32(9): 809-812.

Zoback M L (1992). First and second order patterns of stress in the lithosphere: the world stress map project. J Geophys Res 97(B8): 11 703-11 728. 